\title{
QED vacuum effects in intense laser fields
}

\author{
A. Di Piazza ${ }^{\mathrm{a}}$ and K.Z. Hatsagortsyan ${ }^{\mathrm{b}}$ \\ Max-Planck-Institut für Kernphysik, Saupfercheckweg 1, 69117 Heidelberg, Germany
}

\begin{abstract}
Two quantum electrodynamical processes occurring in the presence of a strong laser field and involving the polarization of quantum vacuum are discussed. First, the process of photon splitting in a laser field is examined and the possibility of its experimental observation is investigated. In particular, it is seen that envisaged progress in the generation of high-flux monochromatic gamma ray beams by employing Compton backscattering by electrons coming from an undulator could lead to an experimental observation of this process. Then, the laser photons merging due to vacuum polarization in the collision of a strong laser field and a proton beam is studied. It is shown that this process allows, in principle, the possibility of observing for the first time non-perturbative refractive vacuum polarization effects primed by a strong laser beam.
\end{abstract}

\section{Introduction}

Perturbative Quantum Electrodynamics (QED) has been tested experimentally with high accuracy (see the recent review [1] and the references therein). On the other hand, testing QED in the presence of strong electromagnetic fields so far has been successful mostly in the case of strong Coulomb fields, i. e. the fields created by highly charged nuclei with charge number $Z \lesssim 1 / \alpha \approx 137[2,3]$. Here, $\alpha=e^{2} / 4 \pi$, with $-e<0$ being the electron charge, is the fine-structure constant (natural units with $\hbar=c=1$ are used throughout this paper). This is due to the fact that the electric field of a highly charged nucleus at the typical QED length $\lambda_{c}=1 / m$ (Compton length) is of the order of the so-called critical electric field $E_{c r}=m^{2} / e=1.3 \times 10^{16} \mathrm{~V} / \mathrm{cm}$, where $m$ is the electron mass. In turn, in the presence of electric fields of the order of $E_{c r}$ the quantum vacuum shows nonlinear dielectric properties as predicted by QED and also spontaneous electron-positron pair creation from vacuum occurs [4]. Another remarkable experimental result has already been obtained at SLAC where electron-positron pair creation was observed for the first time in the collision of an electron beam with an energy of the order of $50 \mathrm{GeV}$ and an optical laser field with an intensity of the order of $10^{19} \mathrm{~W} / \mathrm{cm}^{2}$ [5]. Although the electric field of the laser was several orders of magnitude smaller than $E_{c r}$, the observation of this nonlinear vacuum effect has been possible due to the high energy of the electrons which implies a strong enhancement of the laser electric field in the electrons rest frame. In this respect, the development of laser technology allows the use of intense laser beams to systematically probe the properties of the QED vacuum in the presence of such strong fields [6]. In fact, the typical laser intensity of QED is $I_{c r}=E_{c r}^{2} / 8 \pi=2.3 \times 10^{29} \mathrm{~W} / \mathrm{cm}^{2}$, corresponding to a laser electric field amplitude of $E_{c r}$. Numerous Petawatt laser systems are under construction in different laboratories, and capable, in principle, to attain an intensity of about $10^{23} \mathrm{~W} / \mathrm{cm}^{2}$ [7]. Moreover, the laser at the Extreme Light Infrastructure (ELI) is expected to attain unprecedented intensities of about $10^{25}-10^{26} \mathrm{~W} / \mathrm{cm}^{2}$ [8]. At these intensities other

\footnotetext{
a e-mail: dipiazza@mpi-k.de

b e-mail: k.hatsagortsyan@mpi-k.de
} 


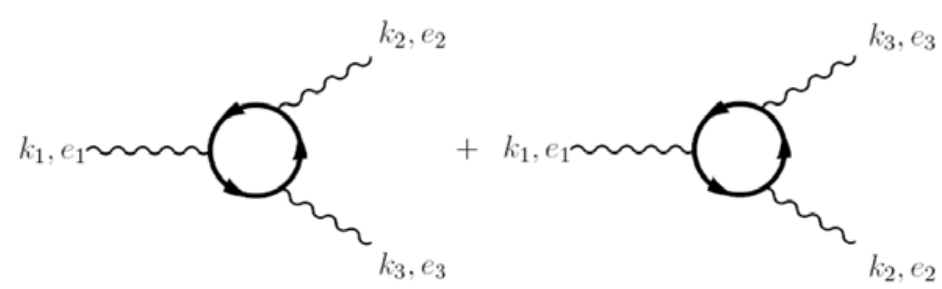

Fig. 1. The Feynman diagrams corresponding to the photon splitting process in a laser field. The thick fermion lines indicate that the dressed fermion propagator in a laser field is employed.

appealing phenomena can be experimentally tested like radiation reaction [9] and the new areas of nuclear quantum optics [10] and laser particle physics [11] can be started to be explored.

In the following we discuss two QED processes occurring in the presence of a strong laser field. First, we study the process of photon splitting in a laser field [12]. This process cannot occur in vacuum due to the conservation of charge-parity in QED and it has already been investigated in the presence of a uniform and static magnetic field [13], in a constant crossed field [14] and in an atomic field [15,16]. Second, we point out the possibility of observing experimentally non-perturbative vacuum polarization effects in the collision of a strong laser field and a high-energy proton beam [17].

\section{Photon splitting in a laser field}

In this section we study the QED process of photon splitting in a laser field [12]. Out of general considerations we expect that the invariant amplitude of this process depends on the laser field parameters only through the two gauge- and Lorentz-invariant quantities $\xi=e E_{0} / m \omega_{0}$ and $\chi=\left(\omega_{1} / m\right)\left(E_{0} / E_{c r}\right)$. Here, it is understood that, without loss of generality, the initial photon with energy $\omega_{1}$ and the laser field with amplitude $E_{0}$ and frequency $\omega_{0}$ are counterpropagating. Now, the total amplitude of this process is schematically represented by the Feynman diagrams in Fig. 1. By applying the Feynman rules to the diagrams in Fig. 1, the amplitude $\mathcal{M}$ of the photon-splitting process is

$$
\mathcal{M}=i e^{3} \int d^{4} x \operatorname{Tr}\left\langle x\left|\mathrm{e}^{-i\left(k_{1} x\right)} \hat{e}_{1} \mathcal{G}[\mathcal{A}] \mathrm{e}^{i\left(k_{2} x\right)} \hat{e}_{2}^{*} \mathcal{G}[\mathcal{A}] \mathrm{e}^{i\left(k_{3} x\right)} \hat{e}_{3}^{*} \mathcal{G}[\mathcal{A}]\right| x\right\rangle+\left(k_{2} \leftrightarrow k_{3}, e_{2} \leftrightarrow e_{3}\right),
$$

where $\mathcal{G}[A]=\left[\gamma^{\mu}\left(i \partial_{\mu}+e A_{\mu}(x)\right)-m+i 0\right]^{-1}$ is the dressed electron propagator in a laser field with four-potential $A^{\mu}(x)$ [12]. As we have mentioned, the laser field is included exactly in the calculations by employing the dressed electron propagator in a laser field. The details of the evaluation of the amplitude $\mathcal{M}$ can be found in [12]. The calculations in the case of a laser field with arbitrary shape and amplitude are, in fact, rather cumbersome. The expressions become significantly simpler when one considers the paradigmatic example of a monochromatic wave. An interesting case is that of a strong optical laser field. In this case, the parameter $\eta=\chi / \xi=\omega_{0} \omega_{1} / m^{2}$ can be assumed to be much smaller than unity and it can be shown that the final photons are almost collinear with the initial one. Moreover, if the wave is circularly polarized, then photon splitting occurs with the absorption of one or three laser photons only. Instead, if the laser field is linearly polarized, then any (odd) number of laser photons can be absorbed. This results from the conservation of the component of the total angular momentum along the common propagation direction of the initial and final photons. The fact that only an odd number of laser photons can be absorbed is a consequence of the Furry theorem [18].

We discuss here the possibility of observing experimentally the photon-splitting process in a strong $\mathrm{x}$-free electron laser (X-FEL) field. The quantity to be measured experimentally is the photon splitting rate $\dot{W}$. This quantity can by calculated from the amplitude $\mathcal{M}$ by applying the usual Fermi golden rule [18]. Even for the intensities which will be available at DESY [19], the parameter $\xi$ will be much smaller than unity (for example, if $\omega_{0}=0.5 \mathrm{keV}$ and $I_{0}=E_{0}^{2} / 8 \pi=10^{15} \mathrm{~W} / \mathrm{cm}^{2}$, then $\left.\xi \sim 10^{-5}\right)$. In this case, the amplitude of photon 
splitting reduces to that of photon-photon scattering. Therefore, it is convenient to choose $\omega_{1}=m^{2} / \omega_{0}=500 \mathrm{MeV}$ for which $\eta=1$. In fact, at $\eta=1$ the cross-section of photonphoton scattering is almost maximal and is approximately given by $\sigma \sim 10^{-30} \mathrm{~cm}^{2}[18]$. Now, it can be shown that with the numerical parameters above one obtains a photon-splitting rate $\dot{W} \approx 60 \mathrm{~s}^{-1}$. The X-FEL at DESY will produce in one second ten trains of pulses, each containing about 4000 pulses of $100 \mathrm{fs}$ of duration. Therefore, the effective interaction time per shot is $4 \times 10^{-10} \mathrm{~s}$. The photon splitting yield depends on the initial photon flux. We underline that the photons in the beam should have a well defined energy. It is very important as the energies of the two final photons are measured at the same time and their sum has to be equal to the initial photon energy (the energy absorbed from the laser field is of course negligible). One way to achieve that is to tag the incoming photons [20]. i.e. to measure the energy of each photon involved in the interaction. In this case, however, the initial photon flux is limited: present technology provides $10^{8}$ tagged photons per second [20]. Then, we only obtain two photon splitting events per hour. Moreover, this estimation is made essentially smaller by the poor spacetime overlapping between the laser and the initial photon beams. In this respect, we conclude that the use of a tagged beam is not suitable for detecting photon splitting in a laser field. Therefore, rather than tagging photons, one should use a nearly monochromatic photon beam. A possible solution could be to use the electrons coming from the undulator of an X-FEL to produce nearly monochromatic high-energy photon beams by means of Compton backscattering, without the necessity of tagging, as suggested in [21]. Photon fluxes up to $10^{11}$ photons per second are expected, leading in the example above to 2000 photon splitting events per hour. Another advantage of this alternative scheme is that the photon beam dimensions would be significantly smaller than those discussed in [20].

\section{QED effects in laser-proton collisions}

In this section we investigate the vacuum polarization effects arising from the collision of a high-energy proton and a strong laser beam. The proton is light enough to be accelerated to very high energies (for example, up to $7 \mathrm{TeV}$ at the Large Hadron Collider (LHC) [22]) in such a way that the laser field in its rest frame is strongly enhanced with respect to its value in the laboratory frame. On the other hand, the proton is heavy enough that the multiphoton Thomson scattering of the laser photons by the proton is negligible. This is important because multiphoton Thomson scattering represents, in principle, a competing process of the process we want to study. In the latter, in fact, laser photons merge into one outgoing photon due to their interaction with the electromagnetic field of the proton mediated by a virtual electronpositron pair. The proton can be modeled as an external field because of its very high energy. The details of the calculation of the photon merging rate can be found in [17]. We consider the case of a relativistic laser field with amplitude $E_{0}$, intensity $I_{0}=E_{0}^{2} / 8 \pi$ and frequency $\omega_{0}$ such that $\xi=e E_{0} / m \omega_{0} \gg 1$. We also assume that the laser beam and the proton are counterpropagating. If $\vartheta$ is the angle between the outgoing photon and the laser propagation direction, the differential rate $d \mathcal{R}_{2 n} / d \vartheta$ relative to the merging of $2 n(n \geq 1)$ laser photons is given by (due to the Furry theorem only an even number of laser photons can merge) [17]

$$
\frac{d \mathcal{R}_{2 n}}{d \vartheta}=\frac{\alpha^{3}}{64 \pi^{2}} \frac{(1+\beta) m^{4}}{\omega_{0}^{3}} \frac{\sin ^{3} \vartheta}{(1-\cos \vartheta)^{4}} \frac{\left|c_{1,2 n}\right|^{2}+\left|c_{2,2 n}\right|^{2}}{n^{3}} .
$$

We have introduced here the proton velocity $\beta$ and the coefficients $c_{j, 2 n}$

$c_{j, 2 n}=e^{-i \pi / 3} \int_{0}^{1} d v \int_{0}^{\infty} \frac{d \lambda}{\lambda} e^{-\exp (i \pi / 3) \lambda-x_{2 n}}\left\{j \chi_{2 n}^{2} \lambda^{2} \frac{1-v^{4 / j}}{16}\left[I_{n}\left(x_{2 n}\right)-I_{n}^{\prime}\left(x_{2 n}\right)\right]+\frac{I_{n}\left(x_{2 n}\right)}{\lambda}\right\}$,

where $j \in\{1,2\}, x_{2 n}=\chi_{2 n}^{2} \lambda^{3}\left(1-v^{2}\right)^{2} / 96$ and where $I_{n}(x)$ and $I_{n}^{\prime}(x)$ are the modified Bessel function of order $n$ and its derivative, respectively. The rate essentially depends only on the parameter $\chi_{2 n}$ given by

$$
\chi_{2 n}=\sqrt{\frac{I_{0}}{I_{c r}}} \frac{2 n(1+\beta) \omega_{0}}{m} \frac{1-\cos \vartheta}{1+\beta \cos \vartheta} .
$$


Below, we provide a numerical example showing the experimental feasibility of the laser photon merging process. We consider the possibility of employing the already operative accelerator Tevatron as a high-energy proton source. The relevant parameters of the proton bunches at the Tevatron are [22]: proton energy $980 \mathrm{GeV}$, number of protons per bunch $24 \times 10^{10}$, bunch transversal radius $29 \mu \mathrm{m}$, bunch length $50 \mathrm{~cm}$. As a strong laser beam, we employ strong attosecond pulses of XUV radiation which have been proposed in [23]. In particular, we use an attosecond pulse of $I_{0}=5.1 \times 10^{22} \mathrm{~W} / \mathrm{cm}^{2}$ intensity with a photon energy of $70 \mathrm{eV}$ and a pulse duration of 60 as. The latter, according to simulations of [23], can be produced by the reflection of a $8 \mathrm{PW}$ Ti:Sapphire laser pulse of $5 \mathrm{fs}$ of duration with a focused spot-radius of $5 \mu \mathrm{m}$ from a plasma surface. In the setup above the average number of photons emitted in one hour as a result of the merging of two and of four laser photons is approximately equal to 900 and 13.8, respectively, and it can be seen that the rate of photons proceeding from two- and four-photon Thomson scattering is safely negligible. The photons resulting from the merging are almost backscattered $(\vartheta \approx \pi)$. The distribution is peaked around $\vartheta_{0}=3.1410$ and at $\vartheta_{0}$ the values of the parameters $\chi_{2}$ and $\chi_{4}$ do not allow the use of the perturbation theory because $\chi_{2}=\chi_{4} / 2 \approx 0.8$. In fact, perturbation theory is allowed only if the parameters $\chi_{2 n}$ are much smaller than unity.

\section{Conclusion}

In this paper we have studied two processes which in principle permit testing QED in the presence of strong laser fields. The first one is photon splitting in a laser field. We have pointed out the experimental difficulties of observing this process by employing initial tagged photon beams. However, the alternative scheme discussed in [21] appears more promising for an experimental detection of the process. The second process we have discussed is the laser photon merging in the collision of a high-energy proton beam with a strong laser field. We have shown that this process is much more feasible than photon splitting in a laser field. Moreover, we have pointed out the possibility of testing QED in a regime where the non-perturbative nature of the vacuum effects primed by the laser field is experimentally observable.

\section{References}

1. S.G. Karshenboim, Phys. Rep. 422, 1 (2005)

2. Sh.Zh. Akhmadaliev, et al., Phys. Rev. C 58, 2844 (1998)

3. Sh.Zh. Akhmadaliev, et al., Phys. Rev. Lett. 89, 061802 (2002)

4. E.S. Fradkin, D.M. Gitman, S.M. Shvartsman, Quantum Electrodynamics with Unstable Vacuum (Springer Verlag, Berlin, 1991)

5. D.L. Burke, et al., Phys. Rev. Lett. 79, 1626 (1997)

6. Y.I. Salamin, et al., Phys. Rep. 427, 41 (2006); G.A. Mourou, T. Tajima, S.V. Bulanov, Rev. Mod. Phys. 78, (2006) 309; M. Marklund, P.K. Shukla, Rev. Mod. Phys. 78, 591 (2006)

7. J. Norby, Laser Focus World, January 1 (2005)

8. See the ELI proposal at http://www.extreme-light-infrastructure.eu/

9. C.H. Keitel, et al., J. Phys. B 31, L75 (1998)

10. T.J. Bürvenich, J. Evers, C.H. Keitel, Phys. Rev. Lett. 96, 142501 (2006)

11. C. Müller, K.Z. Hatsagortsyan, C.H. Keitel, Phys. Lett. B 659, 209 (2008) See also [arXiv:0705.0917] [hep-ph]

12. A. Di Piazza, A.I. Milstein, C.H. Keitel, Phys. Rev. A 76, 032103 (2007)

13. S.L. Adler, et al., Phys. Rev. Lett. 25, 1061 (1970)

14. V.O. Papanyan, V.I. Ritus, Sov. Phys. JETP 34, 1195 (1972)

15. R.N. Lee, A.I. Milstein, V.M. Strakhovenko, Sov. Phys. JETP 85, 1049 (1997)

16. R.N. Lee, et al., Phys. Rep. 373, 213 (2003)

17. A. Di Piazza, K.Z. Hatsagortsyan, C.H. Keitel, Phys. Rev. Lett. 100, 010403 (2008) see also [arXiv: 0708.0475] [hep-ph]

18. V.B. Berestetskii, E.M. Lifshitz, L.P. Pitaevskii, Quantum Electrodynamics, 2nd Ed. (Elsevier, Oxford, 1982) 
19. See the European X-Ray Laser Project XFEL homepage at http://xfel.desy.de/

20. S.J. Hall, et al., Nucl. Instr. Meth. A 368, 698 (1996)

21. V.N. Litvinenko, et al., Phys. Rev. Lett. 78, 4569 (1997)

22. Particle Data Group: W.-M. Yao, et al., J. Phys. G 33, 1 (2006)

23. G. Tsakiris, et al., New J. Phys. 8, 19 (2006) 